\author{
Carolyn M. Hendrickson \\ Bruno Crestani \\ Michael A. Matthay
}

\section{Biology and pathology of fibroproliferation following the acute respiratory distress syndrome}

Received: 19 November 2014

Accepted: 20 November 2014

Published online: 6 December 2014

(C) Springer-Verlag Berlin Heidelberg and ESICM 2014

C. M. Hendrickson · M. A. Matthay (

Departments of Medicine and Anesthesia, Cardiovascular Research Institute, University of California, San Francisco, 505 Parnassus Avenue, Moffitt Hospital, M-917, San Francisco, CA 94143-0624, USA

e-mail: Michael.matthay@ucsf.edu

\section{B. Crestani}

APHP, Hopital Bichat, Service de Pneumologie A, DHU FIRE; University of Paris Diderot; INSERM U1152, Paris, France
The landmark study of low-tidal volume ventilation (LTVV) in acute respiratory distress syndrome (ARDS) demonstrated treatment benefit across a broad patient population [1]. In the era of LTVV, researchers studying ARDS must identify both preventative strategies for ARDS as well as effective treatments for a heterogenous population with a lower mortality rate. Distinguishing among ARDS subphenotypes would improve efficiency in clinical trial design and help clinicians tailor supportive and therapeutic treatments. To this end, there is considerable interest in the use of biomarkers in ARDS to define specific biological phenotypes, describe factors driving resolution of disease and, most importantly, identify patients at highest risk for poor outcomes [2, 3]. This approach to targeted therapy has revolutionized care for breast cancer patients and may facilitate innovative therapies for ARDS [4].
Endotypes have been established by a variety of methods. Some studies rely on measuring specific proteins of interest to establish thresholds that correlate with hard clinical outcomes [3, 5]. Others use sophisticated biostatistical models such as latent class analysis to identify patterns within a dataset that predict response to therapy or outcomes of interest [2]. In an article recently published in Intensive Care Medicine, Forel et al. [6] describe the findings from their biomarker study, "Type III procollagen is a reliable marker of ARDS-associated lung fibroproliferation." The authors identified and validated a threshold level for bronchoalveolar lavage (BAL) concentration of the $\mathrm{N}$-terminal of procollagen peptide III (NT-PCP-III) that correlates with histopathologic fibrosis confirmed on open lung biopsy (OLB), a surrogate outcome presumed to be on the same causal pathway as mortality and prolonged mechanical ventilation, in a subgroup of patients with ARDS who could potentially benefit from therapies that mitigate fibrosis.

While irreversible fibrosis due to ARDS was a common clinical problem before LTVV became the standard of care, the incidence of permanent scarring of lung tissue appears to have declined over the past 15 years. The strongest evidence for the lower incidence is the enrollment difficulty encountered by ARDS Network Researches conducting the Late Steroid Rescue Study (LaSRS) study. Based on earlier disease incidence, the study group anticipated enrolling 400 patients over 3 years. However this plan was revised due to low enrollment, and it ultimately took 7 years to enroll 180 patients from 12 US university medical centers. Furthermore, a large cohort study compared subjects who died between 1991 and 2000 to those who died between 2001 and 2010, and found that among patients at risk for ARDS, the proportion of patients with diffuse alveolar damage (DAD) on autopsy was higher in the first decade of the study [7]. Higher tidal volume ventilation strategies 
Fig. 1 The spectrum of response to the fibroproliferative phase of acute respiratory distress syndrome (ARDS). The fibroproliferative phase of ARDS is followed by a spectrum of host responses that range from resolution of injury and restoration of normal alveolar structure and function to irreversible fibrosis characterized by distorted tissue architecture with fibroblasts in the alveolar septae and distal airways, as well as extensive collagen deposition in the extracellular matrix. Fibroblasts synthesize procollagen, which is cleaved to form collagen that is cross-linked and assembled into collagen fibrils. Forel et al. [6] measured the N-terminal peptide cleaved from procollagen peptide III (NT$P C P-I I I)$ in alveolar lavage fluid as a biomarker for a fibrotic response to the fibroproliferative phase of ARDS that correlates with fibrosis on open lung biopsy and worse clinical outcomes. The illustration was created by Diana Lim
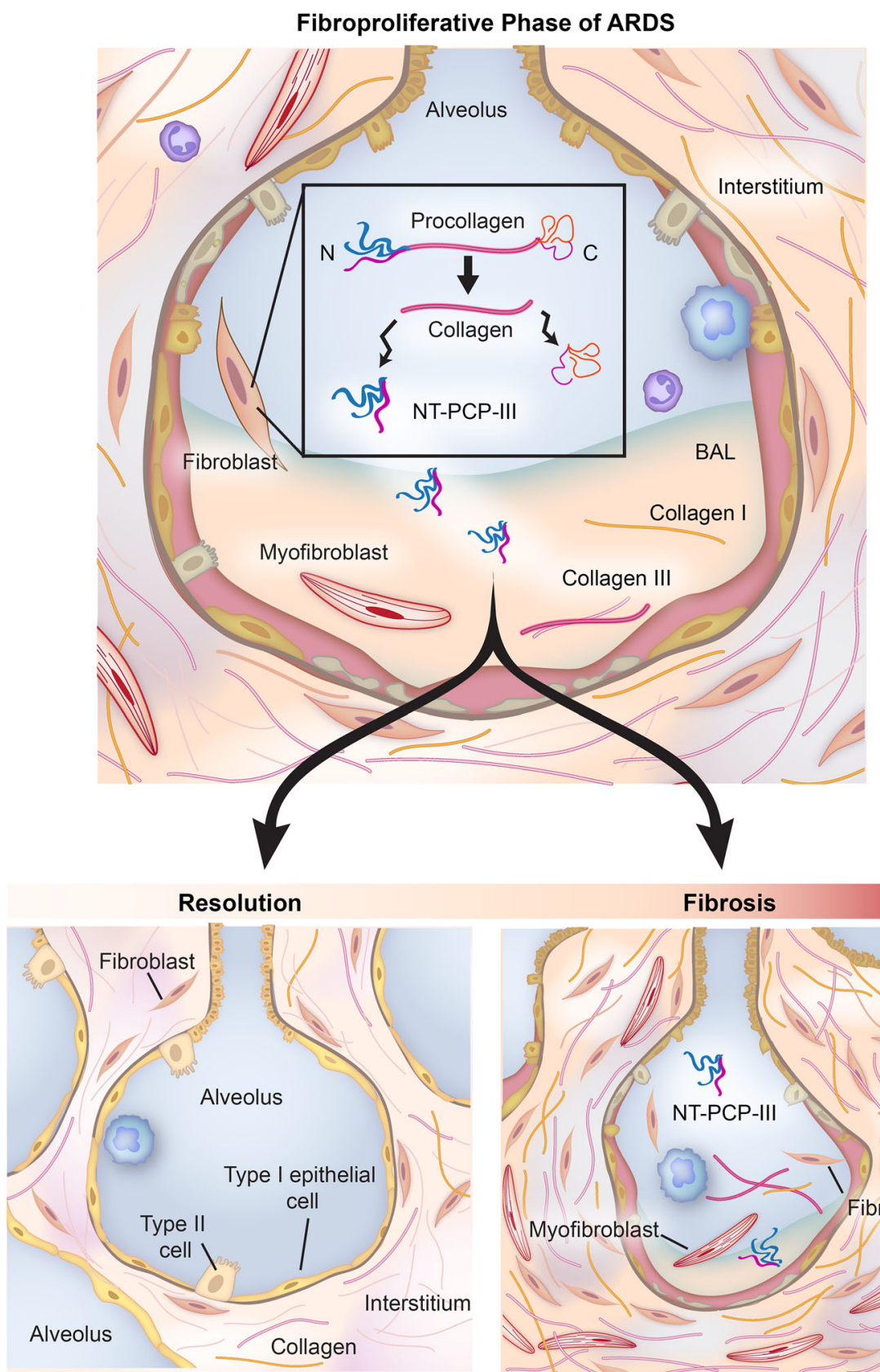

Fibrosis

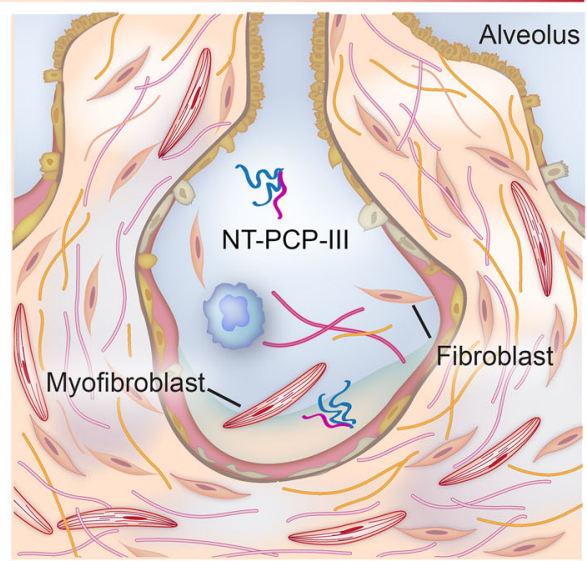

may have driven the incidence of persistent ARDS with fibroproliferative features before 2000 .

Patients who progress to fibrosis after ARDS have prolonged requirements for mechanical ventilation, low lung compliance, and high dead space measurements. However, it is difficult to accurately identify this subgroup of patients using early clinical and demographic characteristics. Even among patients with non-resolving ARDS after 7 days of mechanical ventilation, it is not clear which individuals have irreversible fibrosis without invasive testing with OLB [8]. In the spirit of defining specific ARDS subphenotypes, et al. describe an approach using a biomarker in BAL to identify patients with fibrosis in non-resolving ARDS through a minimally invasive diagnostic test.

NT-PCP-III is the product of protease activity in the extracellular space and a marker of collagen synthesis by pulmonary fibroblasts. Plasma and BAL concentrations of NT-PCP-III in patients with ARDS are elevated in the first $24 \mathrm{~h}$ and correlate with death and ventilator-free days [9-11]. NT-PCP-III is elevated in BAL samples taken $24 \mathrm{~h}$ after ARDS onset, suggesting that fibroblasts are active soon after injury [10]. Furthermore, the concentration of NT-PCP-III in BAL at $24 \mathrm{~h}$ is higher in patients 
who die after ARDS, indicating a shift towards fibrosis after inflammation that sets the stage for a severe pathologic response to injury. Forel et al. [6] show for the first time that the BAL concentration of NT-PCP-III reliably correlates with fibrosis on histopathology specimens from OLB. BAL NT-PCP-III in ARDS is higher in patients who die and have fewer ventilator-free days, despite adherence to LTVV protocols. Overall, the authors present strong evidence that NT-PCP-III may be an important biomarker of fibrosis and might serve as a screening measurement for enrollment in trials of therapies targeted at mitigating fibrosis in patients with non-resolving ARDS.

The data presented by Forel et al. [6] have some limitations. Fibrosis is not strictly defined in the discussion of many of the clinical and translational studies in ARDS. Lung fibrosis is marked by irreversible structural damage to the alveoli and the accumulation of fibroblasts. Fibrosis is preceded by a fibroproliferative or organizing phase characterized by the accumulation of myofibroblastas in a loose extracellular matrix in the alveolar septa and distal air spaces [12]. This fibroproliferative phase is a potentially reversible state and may be the optimal target for corticosteroids in persistent ARDS [13]. A more precise definition of lung abnormalities in lung biopsies is needed in future studies to differentiate earlier fibroproliferation from later fibrosis as sensitivity to glucocorticoid therapy may differ along this spectrum. The inclusion of ten patients from the determination cohort in the validation cohort of 51 subjects is a potential source of bias and may limit the generalizability of the threshold defined by this study. Lastly, the most important limitation of this study is one that is common to many biomarker studies: the authors have only examined one protein from one physiologic compartment at one point in time during a complex and heterogeneous disease process. To fully grasp the enormity of the problem that Forel et al. [6] are tackling, it is important to briefly review what is more generally known about fibroproliferation in ARDS.

Autopsy and histopathological studies in ARDS show an early inflammatory phase followed by a fibroproliferative repair phase, with deposition of matrix proteins, including collagen. Some patients progress to effective tissue repair and resolution of ARDS while others develop irreversible lung fibrosis and have higher mortality rates. As illustrated in Fig. 1, this balance between resolution of injury and ineffective or inappropriate tissue repair is complex and modulated by proinflammatory cytokines, the extracellular matrix, and the effects of aging on host responses [14]. Alveolar fibroblasts are central players in the fibrotic and inflammatory response in ARDS and can be cultured from BAL in $25 \%$ of patients with ARDS, but not from ventilated controls [15]. These cultured fibroblasts behave similarly to fibroblasts isolated from subjects with idiopathic pulmonary fibrosis, exhibiting a blunted response to stimulation with an anti-fibrotic mediators and increased collagen 1 secretion. Not only are the forces driving collagen deposition complex, but for a complete understanding of the physiology and pathology of fibrotic changes in the lung it is important to consider the pathways for degradation of matrix proteins that occur in both normal physiology and disease states [16].

BAL NT-PCP-III may be a valuable biomarker for a subphenotype of ARDS patients with fibroproliferation in the LTVV era. It remains to be established if this biomarker can identify patients who may have a differential response to glucocorticoids or other treatments designed to reduce the lung fibrosis that develops in some patients after ARDS.

Acknowledgments This work was supported in part by NHLBI R37HL51856, R01HL51854, and F32HL124911. The authors thank Diana Lim for help with designing the figure.

\section{References}

1. National Heart L, Blood Institute Acute Respiratory Distress Syndrome Clinical Trials N (2000) Ventilation with lower tidal volumes as compared with traditional tidal volumes for acute lung injury and the acute respiratory distress syndrome. The Acute Respiratory Distress Syndrome Network. N Engl J Med 342(18):1301-1308

2. Calfee CS, Delucchi K, Parsons PE et al (2014) Subphenotypes in acute respiratory distress syndrome: latent class analysis of data from two randomised controlled trials. Lancet Respir Med 2(8):611-620
3. Calfee CS, Ware LB, Glidden DV et al (2011) Use of risk reclassification with multiple biomarkers improves mortality prediction in acute lung injury. Crit Care Med 39(4):711-717

4. Dowsett M, Dunbier AK (2008) Emerging biomarkers and new understanding of traditional markers in personalized therapy for breast cancer. Clin Cancer Res 14(24):8019-8026

5. Fremont RD, Koyama T, Calfee CS et al (2010) Acute lung injury in patients with traumatic injuries: utility of a panel of biomarkers for diagnosis and pathogenesis. J Trauma 68(5):1121-1127
6. Forel JM, Guervilly C, Hraiech $\mathrm{S}$ et al (2014) Type III procollagen is a reliable marker of ARDS-associated lung fibroproliferation. Intensive Care Med. doi:10.1007/s00134-014-3524-0

7. Thille AW, Esteban A, FernandezSegoviano P et al (2013) Comparison of the Berlin definition for acute respiratory distress syndrome with autopsy. Am J Respir Crit Care Med 187(7):761-767

8. Donati SY, Papazian L (2008) Role of open-lung biopsy in acute respiratory distress syndrome. Curr Opin Crit Care 14(1):75-79 
9. Gonzalez-Lopez A, Garcia-Prieto E, Batalla-Solis E et al (2012) Lung strain and biological response in mechanically ventilated patients. Intensive Care Med 38(2):240-247

10. Chesnutt AN, Matthay MA, Tibayan FA, Clark JG (1997) Early detection of type III procollagen peptide in acute lung injury. Pathogenetic and prognostic significance. Am J Respir Crit Care Med 156(31):840-845

11. Clark JG, Milberg JA, Steinberg KP, Hudson LD (1995) Type III procollagen peptide in the adult respiratory distress syndrome. Association of increased peptide levels in bronchoalveolar lavage fluid with increased risk for death. Ann Intern Med 122(1):17-23
12. Castro CY (2006) ARDS and diffuse alveolar damage: a pathologist's perspective. Semin Thorac Cardiovasc Surg 18(1):13-19

13. Lamontagne F, Brower R, Meade M (2013) Corticosteroid therapy in acute respiratory distress syndrome. CMAJ 85(3):216-221

14. White ES, Mantovani AR (2013) Inflammation, wound repair, and fibrosis: reassessing the spectrum of tissue injury and resolution. J Pathol 229(2):141-144
15. Quesnel C, Nardelli L, Piednoir P et al (2010) Alveolar fibroblasts in acute lung injury: biological behaviour and clinical relevance. Eur Respir J 35(6):1312-1321

16. McKleroy W, Lee TH, Atabai K (2013) Always cleave up your mess: targeting collagen degradation to treat tissue fibrosis. Am J Physiol Lung Cell Mol Physiol 304(11):L709-L721 